\title{
Influence of defect in an excited steel plate on the results of time-frequency analysis
}

\author{
Arkadiusz Dobrzycki ${ }^{1, *}$, Anna Knitter-Piątkowska ${ }^{2}$, \\ ${ }^{1}$ Poznan University of Technology, Faculty of Electrical Engineering, Piotrowo 3A, 60-965 Poznan, Poland \\ ${ }^{2}$ Poznan University of Technology, Faculty of Civil Engineering, Piotrowo 5, 60-965 Poznan, Poland
}

\begin{abstract}
The paper pertains the use of non-invasive diagnostic methods to assess the condition of metal elements of machinery and equipment or metal components of building structures. The conducted research concerns the analysis of acoustic signals generated during the excitation of a steel plate and the influence of discontinuities such as grooves and apertures in its structure on spectrogram of recorded acoustic emission signals. Since the presence of the opening causes a significant increase in the initial phase of the acoustic event, higher frequencies (20-55 kHz) and faster decay of the signal, so it should be noted that the frequency analysis of recorded signals, in the initial phase of the acoustic event, can be considered as an indicator of the damage presence.
\end{abstract}

\section{Introduction}

Awareness of the need to monitor the structure (SHM structural health monitoring) and detection of damages at the earliest stage is an issue present in the society of engineers of various specialties. Damage identification methods have been developed for many years. Recently, the role of non-destructive methods (NDT) has significantly increased. The essence of NDT is to locate the damage in a structural element without changing its properties and functionality. Defect detection is the first level of a more general problem namely damage identification. There is a vast variety of methods evaluating the condition of the structure starting from the most basic visual inspection, through radiographic tests, magnetic fields, thermography, modal analysis, which is based on natural frequencies, modal shapes or damping changes and many others. In order to examine the state of technical objects, their models are often used [1,2] and the characteristic values are analyzed $[3,4]$. Another advanced, non-invasive diagnostic methods used in this research work, is the acoustic emission method AE [5-8]. However, the global structural response may be insensitive to local small changes caused by defects, so if one doesn't want to use or doesn't have an information about an intact structure it is advisable to use e.g. wavelet transform, also in its discreet form [9-12].

\section{Results of analysis}

\subsection{Test setup and analysis method}

In order to carry out the experiment, a test stand consisting of an examined (mechanically excited) steel plate $(E=210 \mathrm{GPa})$, a measurement (electroacoustic transducer with amplifying system) and recording signals (measuring card and computer) system was constructed. The exact schematic diagram of measuring unit with the detailed description was presented in [13]. In the very same paper one can find the conclusions from the influence of the substrate different types (on which the plate was placed during tests) and the type of excitation on registered signals frequency spectrum. The performed tests proved that the influence of the substrate, and indirectly its elasticity, does not change the spectrum of diagnostic signals, provided that the plate is induced by an inelastic element. However, the impact of damage on the average spectrum was relatively small, this was the reason for the timefrequency analysis. It is assumed that the frequency distribution may change during the impulse due to e.g. reflections of acoustic waves from the edge of the sample or damage.

The sample was excited at various distances from the transducer from 5 to $25 \mathrm{~cm}$ in increments of $5 \mathrm{~cm}$, five times for each distance. A slight influence of the excitation-transducer distance on the spectrogram was observed.

Registered signals were analyzed while using numerical procedures developed in the Matlab environment. All tests were a real experiment.

\subsection{Sample results}

The individual spectrograms were plotted in relative units, the reference being the largest amplitude among the registered components for each excitation. The obtained spectrograms were compared and it was noted that for a given type of damage, in the analyzed distance 
interval, the distance from damage has little effect on the spectrogram.

Fig. 1 shows a spectrogram for the case of excitation of the undamaged plate, while Fig. 2 shows a spectrogram for a plate with damage in a form of a hole with a $9.5 \mathrm{~mm}$ diameter through the entire thickness, whereas the dimensions of the plate were: 200x300x10 mm

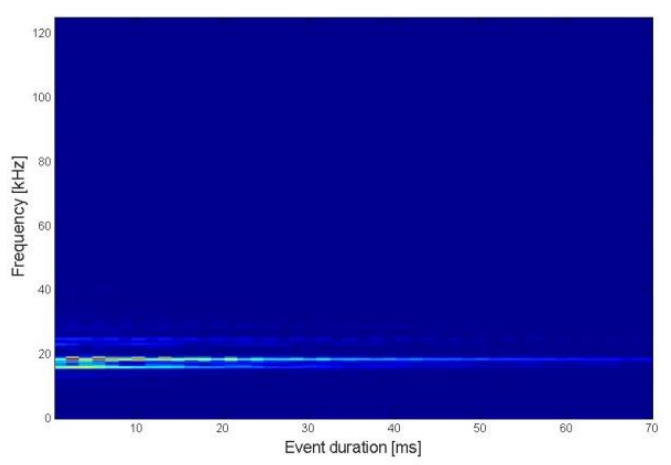

Fig. 1. Spectrogram for the case of excitation of the undamaged plate.

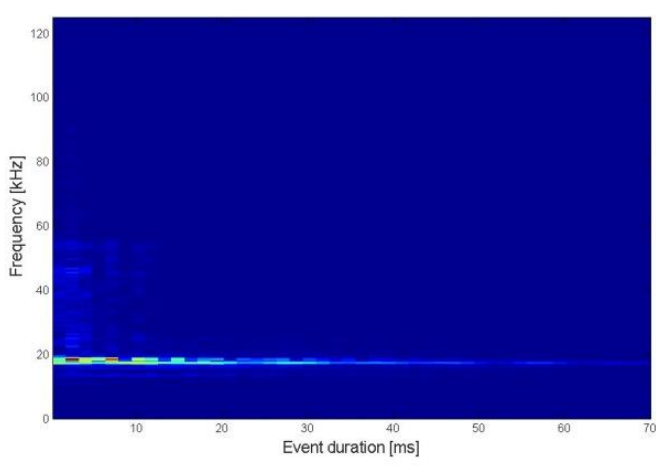

Fig. 2. Spectrogram for a plate with damage in a form of a hole with a $9.5 \mathrm{~mm}$ diameter through the entire thickness.

Analyzing the results presented above, it can be noticed that in both cases: the undamaged and defective element dominates the frequencies in the range of 15-20 $\mathrm{kHz}$. One can, however, notice the clear difference in the initial phase of the analyzed acoustic event. In the case of the presence of damage it results in a much larger, the share of signals with frequencies from the $20-55 \mathrm{kHz}$, range.

It is a confirmation of preliminary frequency analysis of signals carried out for various types of damage [13].

\section{Conclusions}

Precise and quick diagnostics of structural elements is an important practical issue. The use of non-invasive methods reduces the time needed to perform the examinations.

Tests, which were carried out concerned the timefrequency analysis of $\mathrm{AE}$ signals generated during mechanical excitation of the sample, which was a plate made of steel. In the obtained results, it is important to notice that the severity of the damages, meaning superficial groove or apertures through the whole thickness of the element, affects spectra of signal spectrograms [13]. This influence is evident in the distinct frequency band designation from $20-50 \mathrm{kHz}$. However, it should be borne in mind that, while in laboratory tests it is relatively easy to notice this correlation, in real conditions, with an increased level of ambient noise, it may be more difficult. Therefore, it seems reasonable to use other signal parameters or develop the new one, clearly identifying the presence and type of damage as indicators, which is the goal of further work on this topic.

\section{References}

1. L. Kasprzyk, Eksploat. Niezawodn. 19 (2), 229-236 (2017), http://dx.doi.org/10.17531/ein.2017.2.10

2. G. Trzmiel, Eksploat. Niezawodn. 19 (4), 516-521 (2017), http://dx.doi.org/10.17531/ein.2017.4.4

3. J. Jajczyk, A. Dobrzycki, M. Filipiak, D. Kurz, E3S $\begin{array}{llll}\text { Web Conf. } & 19 & 01027 & \text { (2017), }\end{array}$ doi: 10.1051/e3sconf/20171901027

4. K. Bednarek, R. Nawrowski, A. Tomczewski, Electromagnetic Fields in Electrical Engineering, Studies in Applied Electromagnetics and Mechanics, 22, 363-368 (IOS Press, 2002)

5. W. Opydo, A. Dobrzycki, Electr. Eng. 94, 37 (2012) https://doi.org/10.1007/s00202-011-0219-1

6. A. Dobrzycki, S. Mikulski, W. Opydo, 2014 ICHVE International Conference on High Voltage Engineering and Application, 1-4. (2014) doi: 10.1109/ICHVE.2014.7035500

7. A. Dobrzycki, S. Mikulski, Prz. Elektrotech. 92 (4), 221-223 (2016), doi:10.15199/48.2016.04.44

8. A. Dobrzycki, S. Mikulski, Prz. Elektrotech. 92 (4), 26-29 (2016), doi:10.15199/48.2016.04.07

9. S. Mallat, IEEE Trans. Pattern Anal. And Machine Intell., 11, 674-693 (1989)

10. A. Knitter-Piątkowska, M. Guminiak, Advances in Mechanics: Theoretical, Computational and Interdisciplinary Issues, proceedings of the $21^{\text {st }}$ International Conference on Computer Mechanics, red. M. Kleiber, 297-300, (CRC Press, 2016)

11. A. Knitter-Piątkowska, M. Guminiak, G. Hloupis, J. Theor. App. Mech. 55 (2), 481-496 (2017)

12. Z. Pawlak, A. Knitter-Piątkowska, Proceedings of the $22^{\text {nd }}$ International Conference on Computer Mechanics, red. J. Podgórski, 130006-1-130006-9, (AIP Publishing, 2018)

13. A. Dobrzycki, A. Knitter-Piątkowska, Pozn. Univ. Technol. Acad. J. Electr. Eng. 93, 323-334 (2018), doi: 10.21008/j.1897-0737.2018.93.0027 (in polish) 